\title{
New extensions of Rayleigh distribution based on inverted- Weibull and Weibull distributions
}

\author{
Mahmoud M. Smadi, Mahmoud H. Alrefaei \\ Department of Mathematics and Statistics, Jordan University of Science and Technology, Irbid, Jordan
}

\begin{tabular}{l} 
Article Info \\
\hline Article history: \\
Received Sep 4, 2020 \\
Revised Apr 27, 2021 \\
Accepted May 18, 2021 \\
\hline
\end{tabular}

\section{Keywords:}

Estimation

Inverted Weibull distribution

Rayleigh distribution

Reliability

Weibull distribution

\begin{abstract}
The Rayleigh distribution was proposed in the fields of acoustics and optics by lord Rayleigh. It has wide applications in communication theory, such as description of instantaneous peak power of received radio signals, i.e. study of vibrations and waves. It has also been used for modeling of wave propagation, radiation, synthetic aperture radar images, and lifetime data in engineering and clinical studies. This work proposes two new extensions of the Rayleigh distribution, namely the Rayleigh inverted-Weibull (RIW) and the Rayleigh Weibull (RW) distributions. Several fundamental properties are derived in this study, these include reliability and hazard functions, moments, quantile function, random number generation, skewness, and kurtosis. The maximum likelihood estimators for the model parameters of the two proposed models are also derived along with the asymptotic confidence intervals. Two real data sets in communication systems and clinical trials are analyzed to illustrate the concept of the proposed extensions. The results demonstrated that the proposed extensions showed better fitting than other extensions and competing models.
\end{abstract}

This is an open access article under the CC BY-SA license.

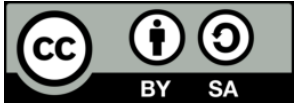

\section{Corresponding Author:}

Mahmoud M. Smadi

Department of Mathematics and Statistics

Jordan University of Science and Technology

Irbid, Jordan

Email: smadi@just.edu.jo

\section{INTRODUCTION}

Probability distributions have been popularly used as models to describe and predict real world phenomena in different fields such as engineering, medical sciences, and biological studies. The statistical analysis depends heavily on the assumed model. In recent years, there have been many interests among statisticians on proposing generalizations and extensions of some well-known probability distributions to provide more flexibility in modeling data. These extensions are based on transformations and compounding by introducing one or more additional parameter (s) to the baseline distribution. Different generated families of distributions are proposed in the literature. Some of these generated families include exponentiated distributions [1]-[3], Kumaraswamy generalized family of distributions [4], Marshall and Olkin family distributions [5], quadratic transmutation map method to generate new probability distributions [6], transformed-transmuter method [7], alpha power transformation method [8], the type I half-logistic family of distributions [9], type II half logistic family of distributions with applications [10], generalized betagenerated distributions [11], and Weibull-G family of probability distributions [12].

The Rayleigh distribution is one of the most popular distributions in analyzing skewed data. It has wide applications in communication systems and modeling lifetime data. To add flexibility to the Rayleigh distribution to get the best model fitting, various generalizations of the distribution have been derived and 
studied. Surles and Padgett [13] considered the two parameter burr type X distribution by introducing a shape parameter and correctly naming it as the generalized Rayleigh (GR) distribution. Gomesa et al. [14] proposed a new distribution called Kumaraswamy generalized Rayleigh distribution. Kadim and Mohammed [15] proposed and studied a new generalization called Rayleigh Pareto distribution. Kundu and Raqab [16] proposed generalized Rayleigh (GR) distribution. Saudi and Sohail [17] proposed modified Rayleigh distribution (MR). Abdulhakim [18] studied a new extended Rayleigh distribution. This paper proposes and considers two generalizations of Rayleigh distribution known as Rayleigh inverted-Weibull (RIW) and Rayleigh Weibull (RW) distributions based on transformed transformer method by Alzaatreh et al. [7]. Such fundamental properties as reliability and hazard functions, moments, quantile function, random number generation, skewness and kurtosis, maximum likelihood estimators were studied with applications on real data sets.

\section{RAYLEIGH INVERTED-WEIBULL DISTRIBUTION}

Suppose $\mathrm{X}$ is a random variable following Rayleigh distribution. Then its probability density function (pdf), cumulative distribution function (cdf), and reliability function are respectively given by:

$$
\begin{aligned}
& f(x)=2 \beta^{2} x e^{-(\beta x)^{2}} x \geq 0, \beta>0, \\
& F(x)=1-e^{-(\beta x)^{2}}, x \geq 0, \beta>0, \\
& r(x)=e^{-(\beta x)^{2}} .
\end{aligned}
$$

Alzaatreh et al. [7] propose transformed transformer method for generating families of continuous distributions, among these new families:

$$
1-F(x)=\int_{0}^{-\log (G(x))} f(t) d t
$$

where $f(t)$ is the baseline distribution, $G(x)$ is the cumulative distribution function of certain continuous distribution.

This new method for generating new distribution has been used by many authors. For example, Ganji et al. [19] introduced the Weibull-Rayleigh distribution, and Kadim and Mohammed [15] introduced Rayleigh Pareto distribution. Bahati et al. [20] studied the properties and applications of Lindley-exponential distribution. Nosakhare and Festus [21] proposed three parameter generalized Lindley distribution. In (2), if the baseline distribution $f(t)$ is the Rayleigh distribution given in (1), then (2) becomes;

$$
1-F(x)=\int_{0}^{-\log (G(x))} 2 \beta^{2} t e^{-(\beta t)^{2}} d t
$$

If we consider $G(x)$ as the one parameter inverted Weibull distribution where its cumulative distribution function (cdf) is given by:

$$
G(x)=e^{-x^{-\alpha}} \cdot x \geq 0, \alpha>0
$$

In (3) becomes;

$$
1-F(x)=\int_{0}^{x^{-\alpha}} 2 \beta^{2} t e^{-(\beta t)^{2}} d t .
$$

The cumulative distribution function (cdf) of the extended distribution known as Rayleigh inverted Weibull distribution is given by:

$$
F(x)=e^{-\beta^{2} x^{-2 \alpha}} x \geq 0, \alpha>0, \beta>0
$$

The probability density function (pdf) is given by:

$$
f(x)=2 \alpha \beta^{2} x^{-2 \alpha-1} e^{-\beta^{2} x^{-2 \alpha}} x>0, \alpha>0, \sigma^{2}>0
$$

The probability density functions (pdf) of Rayleigh inverted Weibull (RIW) distribution with different values of $\alpha$ and $\beta=1$ are shown in Figure 1 which demonstrates the flexibility of the RIW distribution. The pdf is unimodal, right skewed and becomes less peaked with increasing values of $\alpha$ as shown in Figure 1 . 


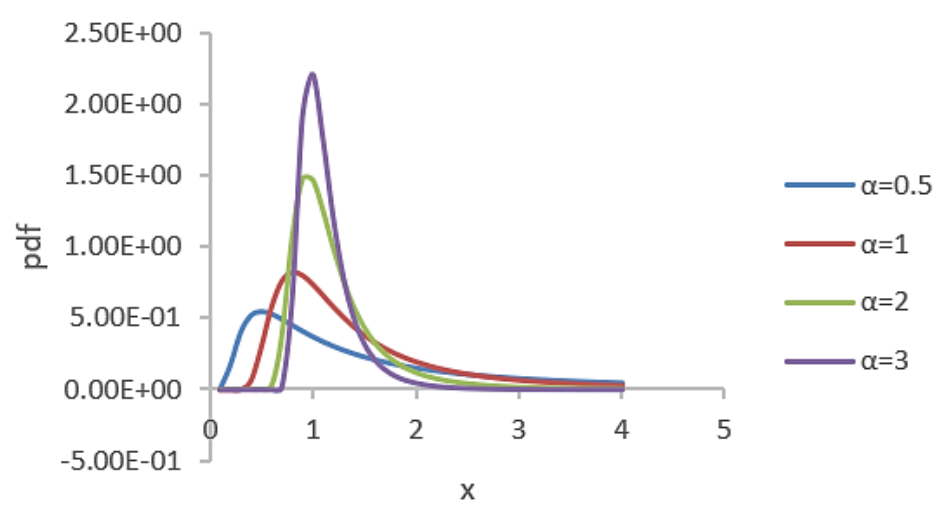

Figure 1. The pdf of RIW distribution with $\alpha=0.5,1,2,3$ and $\beta=1$

\section{RAYLEIGH WEIBULL (RW) DISTRIBUTION}

Alzaatreh et al. [7] proposed transformed transformer method for generating families of continuous distributions, among these new families:

$$
1-F(x)=\int_{0}^{-\log (1-G(x))} f(t) d t
$$

where $f(t)$ is the baseline distribution, and $G(x)$ is the cumulative distribution function of certain continuous distribution called transformed transmuter distribution.

In (6), if the baseline distribution $f(t)$ is the Rayleigh distribution given in (1), then (6) becomes.

$$
F(x)=\int_{0}^{-\log (1-G(x))} 2 \beta^{2} t e^{-(\beta t)^{2}} d t .
$$

Considering $G(x)$ as the one parameter Weibull distribution where its cumulative distribution function (cdf) is given by:

$$
G(x)=1-e^{-x^{\alpha}}
$$

In (7) becomes.

$$
\begin{aligned}
& F(x)=\int_{0}^{-\log (1-G(x))} 2 \beta^{2} t e^{-(\beta t)^{2}} d t \\
& =\int_{0}^{x^{\alpha}} 2 \beta^{2} t e^{-(\beta t)^{2}} d t
\end{aligned}
$$

The cumulative distribution function (cdf) of the extended distribution known as Rayleigh Weibull distribution is given by:

$$
F(x)=1-e^{-\beta^{2} x^{2 \alpha}}
$$

The probability density function (pdf) is given by:

$$
f(x)=2 \alpha \beta^{2} x^{2 \alpha-1} e^{-\beta^{2} x^{2 \alpha}} .
$$

The probability density functions (pdf) of Rayleigh Weibull (RW) distribution with $\beta=1$ and different values of $\alpha$ are shown in Figure 2, which demonstrates the flexibility of the RW distribution. The pdf is unimodal, right skewed and becomes less peaked with increasing values of $\alpha$, for values of $\alpha$ less than one the pdf is a decreasing function, see Figure 2. 


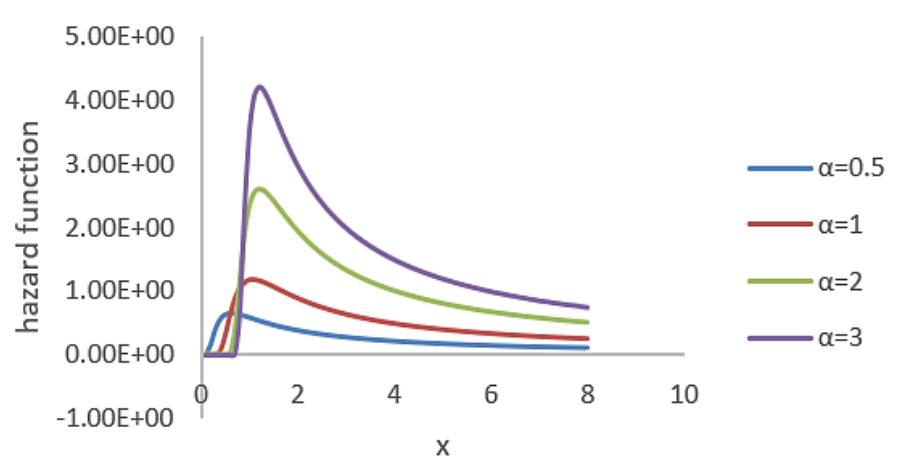

Figure 2. The pdf of RW distribution for $\alpha=0.5,1,2,3$ and $\beta=1$

\section{PROPERTIES OF RAYLEIGH INVERTED-WEIBULL DISTRIBUTION}

\subsection{Reliability and hazard functions}

The survival function, $r(x)$ and hazard function, $h(x)$ of the Rayleigh inverted Weibull distribution are given by:

$$
r(x)=1-e^{-\beta^{2} x^{-2 \alpha}}, h(x)=\frac{2 \alpha \beta^{2} x^{-2 \alpha-1} e^{-\beta^{2} x^{-2 \alpha}}}{1-e^{-\beta^{2} x^{-2 \alpha}}} .
$$

The hazard function of Rayleigh inverted-weibull (RIW) distributions with $\beta=1$ and different values of $\alpha$ are shown in Figure 3, which indicates that the hazard rate function is increasing and decreasing, right skewed and becomes large peaked with increasing values of $\alpha$.

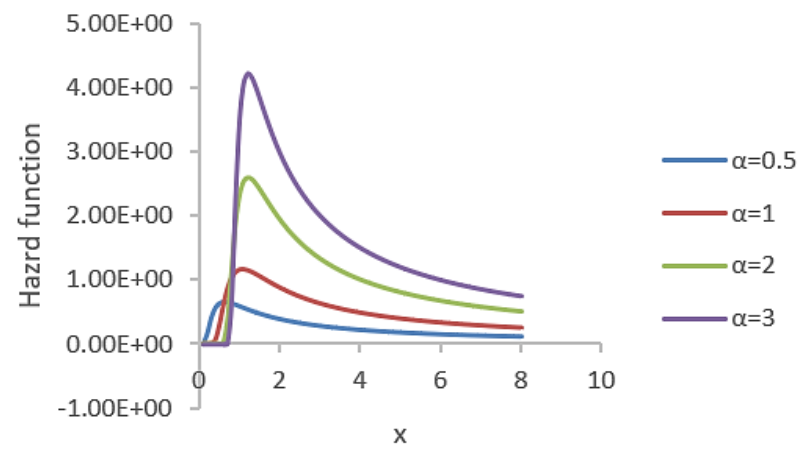

Figure 3. The hazard rate function of RW distribution with $\beta=1$ and $\alpha=0.5,1,2,3$

\subsection{Moments}

- Theorem (1)

The $k^{\text {th }}$ moment of (RIW) distribution is given by:

$$
E\left(X^{k}\right)=2 \alpha \beta^{\frac{k-1}{\alpha}} \Gamma\left(\frac{1+2 \alpha-k}{2 \alpha}+1\right) ; k=1,2,3, \ldots
$$

- Proof:

$$
\begin{aligned}
& E\left(X^{k}\right)=\int_{0}^{\infty} x^{k} f(x) d x \\
& E\left(X^{k}\right)=\int_{0}^{\infty} x^{k} 2 \alpha \beta^{2} x^{-2 \alpha-1} e^{-\beta^{2} x^{-2 \alpha}} d x E\left(X^{k}\right)=2 \alpha \beta^{2} \int_{0}^{\infty} x^{k-2 \alpha-1} e^{-\beta^{2} x^{-2 \alpha}} d x \\
& \text { Let } t=\beta^{2} x^{-2 \alpha}, \text { then } x=\left(\frac{\beta^{2}}{t}\right)^{1 / 2 \alpha}, \text { so }
\end{aligned}
$$




$$
\begin{aligned}
& E\left(X^{k}\right)=2 \alpha \beta^{2} \int_{0}^{\infty}\left(\frac{\beta^{2}}{t}\right)^{\frac{k-2 \alpha-1}{2 \alpha}} e^{-t} d x E\left(X^{k}\right) \\
& =2 \alpha \beta^{\frac{k-1}{\alpha}} \int_{0}^{\infty} t^{\frac{1+2 \alpha-k}{2 \alpha}} e^{-t} d x E\left(X^{k}\right) \\
& =2 \alpha \beta^{\frac{k-1}{\alpha}} \Gamma\left(\frac{1+2 \alpha-k}{2 \alpha}+1\right) .
\end{aligned}
$$

- Mean and variance:

The mean and variance of RF distribution are, respectively given by:

$$
\begin{aligned}
& E(X)=2 \alpha \\
& \operatorname{Var}(X)=\left(2 \alpha \beta^{1 / \alpha} \Gamma\left(\frac{2 \alpha-1}{2 \alpha}+1\right)\right)^{2}-4 \alpha^{2} .
\end{aligned}
$$

\subsection{Quantile and random number generation}

The $q^{\text {th }}$ quantile of Rayleigh inverted Weibull (RIW) distribution is given by:

$$
x_{p}=Q(p)=F^{-1}(p)=\left(\frac{-\beta^{2}}{\log p}\right)^{1 / 2 \alpha}, 0<p<1 .
$$

The median of the distribution is.

$$
x_{\frac{1}{2}}=\left(\frac{-\beta^{2}}{\log \frac{1}{2}}\right)^{1 / 2 \alpha}
$$

The random number generator of Rayleigh inverted Weibull distribution is given by:

$$
x=\left(\frac{-\beta^{2}}{\log u}\right)^{1 / 2 \alpha}
$$

where $\mathrm{u}$ is the random number from uniform distribution; $\mathrm{U}(0,1)$.

\subsection{Skewness and Kurtosis} quantipes:

Because the moments involve gamma function, the kurtosis and skewness can be found using

$$
\begin{aligned}
\text { Skewness }= & \frac{\left(Q_{3}-Q_{2}\right)-\left(Q_{2}-Q_{1}\right)}{\left(Q_{3}-Q_{2}\right)+\left(Q_{2}-Q_{1}\right)} \\
\text { Skewness }= & \frac{\left(\sqrt[2 \alpha]{\frac{-\beta^{2}}{\log 0.75}}-\sqrt[2 \alpha]{\frac{-\beta^{2}}{\log 0.5}}\right)-\left(\sqrt[2 \alpha]{\frac{-\beta^{2}}{\log 0.5}}-\sqrt[2 \alpha]{\frac{-\beta^{2}}{\log 0.25}}\right)}{\left(\sqrt[2 \alpha]{\frac{-\beta^{2}}{\log 0.75}}-\sqrt[2 \alpha]{\frac{-\beta^{2}}{\log 0.5}}\right)+\left(\sqrt[2 \alpha]{\frac{-\beta^{2}}{\log 0.5}}-\sqrt[2 \alpha]{\frac{-\beta^{2}}{\log 0.25}}\right)}
\end{aligned}
$$

A robust alternative measure of kurtosis using quantiles suggested by Moors [22] is given by:

$$
\text { Kurtosis }=\frac{\left(E_{7}-E\right)_{5}-\left(E_{3}-E_{1}\right)}{\left(E_{6}-E_{2}\right)},
$$

where $E_{\mathrm{i}}$ is the $i^{\text {th }}$ octile $E_{i}=F^{-1}(i / 8)$. 


\section{PROPERTIES OF RAYLEIGH WEIBULL DISTRIBUTION}

\subsection{The reliability and hazard functions}

The survival function $r(x)$ and hazard function $h(x)$ of the Rayleigh Weibull distribution are given by

$$
r(x)=e^{-\beta^{2} x^{2 \alpha}}, h(x)=2 \alpha \beta^{2} x^{2 \alpha-1} .
$$

The hazard function of Rayleigh Weibull (RW) distribution with $\beta=1$ and $\alpha=0.7$ and $\alpha=2$ are shown in Figure 4. It is noted that the hazard rate function is increasing concave down trend for $\alpha=0.7$ and increasing concave up trend for $\alpha=2$, it is also noted for values of $\alpha$ greater than one, the hazard function is increasing concave up trend.
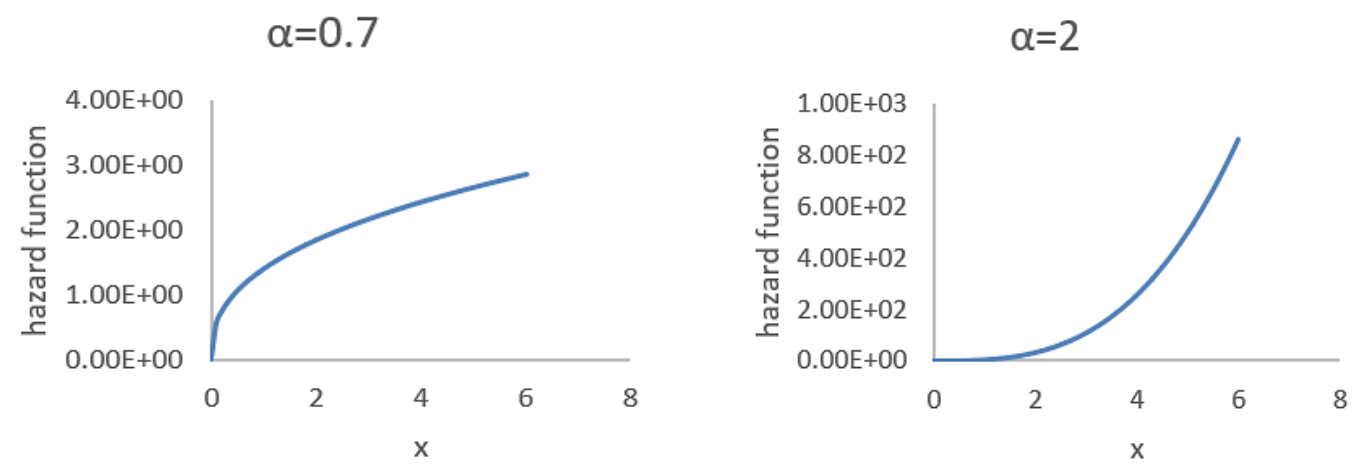

Figure 4. The hazard rate function of RW distribution with $\beta=1$ and $\alpha=0.7$ and $\alpha=2$

\subsection{Moments}

- Theorem (2)

The $k^{\text {th }}$ moment of Rayleigh Weibull distribution about the origin is given by:

$$
E\left(X^{k}\right)=2 \alpha \beta^{\frac{1-k}{\alpha}} \Gamma\left(\frac{k+2 \alpha-1}{2 \alpha}+1\right)
$$

- Proof:

$$
E\left(X^{k}\right)=\int_{0}^{\infty} x^{k} f(x) d x=\int_{0}^{\infty} x^{k} 2 \alpha \beta^{2} x^{2 \alpha-1} e^{-\beta^{2} x^{2 \alpha}} d x=2 \alpha \beta^{2} \int_{0}^{\infty} x^{k+2 \alpha-1} e^{-\beta^{2} x^{2 \alpha}} d x
$$

Let $t=\beta^{2} x^{2 \alpha}$, then $x=\left(\frac{t}{\beta^{2}}\right)^{1 / 2 \alpha}$. Therefore

$$
E\left(X^{k}\right)=2 \alpha \beta^{2} \int_{0}^{\infty}\left(\frac{t}{\beta^{2}}\right)^{\frac{k+2 \alpha-1}{2 \alpha}} e^{-t} d x=2 \alpha \beta^{\frac{1-k}{\alpha}} \Gamma\left(\frac{k+2 \alpha-1}{2 \alpha}+1\right) .
$$

- Mean and variance.

The mean and variance of RF distribution are, respectively given by $E(X)=2 \alpha$

$$
\operatorname{Var}(X)=\left(2 \alpha \beta^{-\frac{1}{\alpha}} \Gamma\left(\frac{1+4 \alpha}{2 \alpha}\right)\right)^{2}-4 \alpha^{2}
$$

\subsection{Quantile and random number generation}

The $q^{\text {th }}$ quantile of Rayleigh Weibul (RW) is given by:

$$
x_{p}=Q(p)=F^{-1}(p)=\left(-\frac{\log (1-p)}{\beta^{2}}\right)^{1 / 2 \alpha}
$$


where $\mathrm{F}^{-1}($.$) is the inverse distribution function.$

The median of the distribution is given by:

$$
x_{\frac{1}{2}}=\left(-\frac{\log (1 / 2)}{\beta^{2}}\right)^{1 / 2 \alpha}
$$

The random number generator of Rayleigh Frechet distribution can be expressed as:

$$
x=\left(-\frac{\log (1-u)}{\beta^{2}}\right)^{1 / 2 \alpha}
$$

where $\mathrm{u}$ is the random number from uniform distribution $\mathrm{U}(0,1)$.

\subsection{Skewness and Kurtosis}

Because the moments involve gamma function, kurtosis and skewness can be found using quantiles:

$$
\begin{aligned}
\text { Skewness }= & \frac{\left(Q_{3}-Q_{2}\right)-\left(Q_{2}-Q_{1}\right)}{\left(Q_{3}-Q_{2}\right)+\left(Q_{2}-Q_{1}\right)}, \text { therefore } \\
\text { Skewness }= & \frac{\left(\sqrt[2 \alpha]{-\frac{\log (0.25)}{\beta^{2}}}-\sqrt[2 \alpha]{-\frac{\log (0.5)}{\beta^{2}}}\right)-\left(\sqrt[2 \alpha]{-\frac{\log (0.5)}{\beta^{2}}}-\sqrt[2 \alpha]{-\frac{\log (0.75)}{\beta^{2}}}\right)}{\left(\sqrt[2 \alpha]{-\frac{\log (0.25)}{\beta^{2}}}-\sqrt[2 \alpha]{-\frac{\log (0.5)}{\beta^{2}}}\right)+\left(\sqrt[2 \alpha]{-\frac{\log (0.5)}{\beta^{2}}}-\sqrt[2 \alpha]{-\frac{\log (0.75)}{\beta^{2}}}\right)},
\end{aligned}
$$

The kurtosis measure is given by;

$$
\text { Kurtosis }=\frac{\left(E_{7}-E\right)_{5}-\left(E_{3}-E_{1}\right)}{\left(E_{6}-E_{2}\right)} \text {, Where } E_{\mathrm{i}} \text { is the } i^{\text {th }} \text { octile } E_{i}=F^{-1}(i / 8) \text {. }
$$

\section{ESTIMATION OF RAYLEIGH INVERTED-WEIBULL DISTRIBTION}

In this section, the estimates of the parameters of the Rayleigh inverted Weibull distribution are derived. Let $X_{1}, X_{2}, \ldots, X_{n}$ be a random sample from Rayleigh inverted Weibull distribution with probability density function given in (5). Then the likelihood function is given by:

$$
L=\prod_{i=1}^{n} 2 \alpha \beta^{2} x_{i}^{-2 \alpha-1} e^{-\beta^{2} x_{i}^{-2 \alpha}}=2^{n} \alpha^{n} \beta^{2 n}\left[\prod_{i=1}^{n} x_{i}^{-2 \alpha-1}\right] e^{-\beta^{2} \sum_{i=1}^{n} x_{i}^{-2 \alpha}} .
$$

The ln-likelihood function can be expressed as:

$$
L=n \log 2+n \log \alpha+2 n \log \beta-(2 \alpha+1) \sum_{i=1}^{n} \ln x_{i}-\beta^{2} \sum_{i=1}^{n} x_{i}^{-2 \alpha}
$$

The normal equations become:

$$
\begin{aligned}
& \frac{\partial \ln L}{\partial \alpha}=\frac{n}{\alpha}-2 \sum_{i=1}^{n} \ln x_{i}+2 \beta^{2} \sum_{i=1}^{n} x_{i}^{-2 \alpha}=0, \\
& \frac{\partial \ln L}{\partial \beta}=\frac{2 n}{\beta}-2 \beta \sum_{i=1}^{n} x_{i}^{-2 \alpha}=0,
\end{aligned}
$$

Which implies that

$$
\beta=\sqrt{\frac{n}{\sum_{i=1}^{n} x_{i}^{-2 \alpha}}}
$$


The following equation can be obtained by substituting (11) in (10).

$$
g(\alpha)=\frac{n}{\alpha}-2 \sum_{i=1}^{n} \ln x_{i}+2 n \frac{\sum_{i=1}^{n} x_{i}^{-2 \alpha} \log \left(x_{i}\right)}{\sum_{i=1}^{n} x_{i}^{-2 \alpha}}=0 .
$$

Therefore, MLE of $\alpha$, can be obtained by solving $g(\alpha)$ using numerical methods, once the MLE for $\alpha$ is obtained, the MLE for $\beta$ can be concluded from (11). The asymptotic normality results can be stated to obtain the approximate confidence intervals for the maximum likelihood estimators. The Fisher information matrix $I(\beta, \alpha)$ can be written is being as.

$$
I(\beta, \alpha)=\left[\begin{array}{c}
-\frac{\partial^{2} \log L(\beta, \alpha)}{\partial \beta}-\frac{\partial^{2} \log L(\beta, \alpha)}{\partial \beta \partial \alpha} \\
-\frac{\partial^{2} \log L(\beta, \alpha)}{\partial \beta \partial \alpha}-\frac{\partial^{2} \log L(\beta, \alpha)}{\partial \alpha^{2}}
\end{array}\right] .
$$

The elements of the observed fisher information matrix are being as.

$$
\begin{aligned}
& \frac{\partial^{2} \log L(\beta, \alpha)}{\partial \beta^{2}}=-\frac{2 n}{\beta^{2}}-2 \sum_{i=1}^{n} x_{i}^{-2 \alpha}, \\
& \frac{\partial^{2} \log L(\beta, \alpha)}{\partial \alpha^{2}}=-\frac{n}{\alpha^{2}}-4 \beta^{2} \sum_{i=1}^{n} x_{i}^{-2 \alpha} \ln x_{i}, \\
& \frac{\partial^{2} \log L(\beta, \alpha)}{\partial \beta \partial \alpha}=4 \beta \sum_{i=1}^{n} x_{i}^{-2 \alpha} \ln x_{i} .
\end{aligned}
$$

The asymptotic distribution of MLEs is a bivariate normal that can be expressed as:

$$
\left(\begin{array}{l}
\hat{\beta} \\
\hat{\alpha}
\end{array}\right) \approx B V N\left(\left(\begin{array}{l}
\beta \\
\alpha
\end{array}\right), \mathrm{I}^{-1}(\hat{\beta}, \hat{\alpha})\right) \text {. }
$$

Therefore, the two sided $100(1-\alpha) \%$ asymptotic cl approximate intervals for $\sigma 2$ and $\alpha$ are:

$$
\hat{\beta} \pm z_{\frac{\alpha}{2}} \sqrt{\operatorname{var}(\hat{\beta})}, \hat{\alpha} \pm z_{\frac{\alpha}{2}} \sqrt{\operatorname{var}(\hat{\alpha})}
$$

where $z \alpha / 2$ is the upper $(\alpha / 2)$-th percentile of the standard normal distribution.

\section{ESTIMATION OF RAYLEIGH WEIBULL DISTRIBUTION}

In this section, the estimates of the parameters of the Rayleigh Weibull distribution are derived. Let $X_{1}, X_{2}, \ldots, X_{n}$ be a random sample from the Rayleigh Weibull distribution with probability density function given in (3.9). Then the likelihood function is given by:

$$
L=\prod_{i=1}^{n} 2 \alpha \beta^{2} x^{2 \alpha-1} e^{-\beta^{2} x^{2 \alpha}}=2^{n} \alpha^{n} \beta^{2 n}\left[\prod_{i=1}^{n} x_{i}^{2 \alpha-1}\right] e^{-\beta^{2} \sum_{i=1}^{n} x_{i}^{2 \alpha}}
$$

The ln-likelihood function is given by:

$$
L=2 \log n+n \log \alpha+2 n \log \beta+(2 \alpha-1) \sum_{i=1}^{n} \ln x_{i}-\beta^{2} \sum_{i=1}^{n} x_{i}^{2 \alpha} .
$$

The normal equations become: 


$$
\begin{aligned}
& \frac{\partial \ln L}{\partial \alpha}=\frac{n}{\alpha}+2 \sum_{i=1}^{n} \ln x_{i}-2 \beta^{2} \sum_{i=1}^{n} x_{i}^{2 \alpha} \ln x_{i}=0, \\
& \frac{\partial \ln L}{\partial \beta}=\frac{2 n}{\beta}-2 \beta \sum_{i=1}^{n} x_{i}^{2 \alpha}=0 .
\end{aligned}
$$

Which implies that

$$
\beta=\sqrt{\frac{n}{\sum_{i=1}^{n} x_{i}^{2 \alpha}}}
$$

Substituting (13) in (12), we obtain

$$
g(\alpha)=\frac{n}{\alpha}+2 \sum_{i=1}^{n} \ln x_{i}-2 n \frac{\sum_{i=1}^{n} x_{i}^{2 \alpha} \ln x_{i}}{\sum_{i=1}^{n} x_{i}^{2 \alpha}}=0 .
$$

Therefore, MLE of $\alpha$, can be obtained by solving $g(\alpha)$ using numerical methods, once the MLE for $\alpha$ is obtained, the MLE for $\beta$ can be conclude from (7.2). The asymptotic normality results can be stated to obtain the approximate confidence intervals for the maximum likelihood estimators. The Fisher information matrix $I\left(\alpha^{2}, \alpha\right)$ can be written is being as:

$$
I(\beta, \alpha)=\left[\begin{array}{cc}
-\frac{\partial^{2} \log L(\beta, \alpha)}{\partial \beta}-\frac{\partial^{2} \log L(\beta, \alpha)}{\partial \beta \partial \alpha} \\
-\frac{\partial^{2} \log L(\beta, \alpha)}{\partial \beta \partial \alpha}-\frac{\partial^{2} \log L(\beta, \alpha)}{\partial \alpha^{2}}
\end{array}\right] .
$$

The elements of the observed Fisher information matrix are being as;

$$
\begin{aligned}
& \frac{\partial^{2} \log L(\beta, \alpha)}{\partial \beta^{2}}=-\frac{2 n}{\beta^{2}}-2 \sum_{i=1}^{n} x_{i}^{2 \alpha}, \\
& \frac{\partial^{2} \log L(\beta, \alpha)}{\partial \alpha^{2}}=-\frac{n}{\alpha^{2}}-4 \beta^{2} \sum_{i=1}^{n} x_{i}^{2 \alpha} \ln x_{i}, \\
& \frac{\partial^{2} \log L(\beta, \alpha)}{\partial \beta \partial \alpha}=4 \beta \sum_{i=1}^{n} x_{i}^{2 \alpha} \ln x_{i} .
\end{aligned}
$$

The asymptotic distribution of MLEs is a bivariate normal that can be expressed as:

$$
\left(\begin{array}{l}
\hat{\beta} \\
\hat{\alpha}
\end{array}\right) \approx B V N\left(\left(\begin{array}{l}
\beta \\
\alpha
\end{array}\right), \mathrm{I}^{-1}(\hat{\beta}, \hat{\alpha})\right) .
$$

Therefore, the two sided 100(1- $\alpha) \%$ asymptotic cl approximate intervals for $\beta$ and $\alpha$ are;

$$
\hat{\beta} \pm Z_{\frac{\alpha}{2}} \sqrt{\operatorname{var}(\hat{\beta})}, \hat{\alpha} \pm Z_{\frac{\alpha}{2}} \sqrt{\operatorname{var}(\hat{\alpha})}
$$

\section{APPLICATIONS}

\subsection{Application 1}

The following data set presents repair times (in $\mathrm{h}$ ) for an airborne communication transceiver. This data set has been previously analyzed by Saudi and Sohail [17]. The data consists of 45 values and are displayed in Table 1. The authors analyzed and compared the proposed models Rayleigh inverted-Weibull (RIW) and Rayleigh Weibull distributions (RW) with other extensions and competing models. Exponential,

New extensions of Rayleigh distribution based on inverted-Weibull and Weibul ... (Mahmoud M. Smadi) 
Rayleigh, generalized exponential (GE) [23], generalized Rayleigh (GR) [16], modified Rayleigh (MR) [17] are considered by the authors, their results are reported in Table 2. The Kolmogorov Simonov test is used to assess if this data set fits these distributions. The theoretical critical value of Kolmogorov Smirnov (K-S) test is $K-S_{0.05}=0.2027$ at 0.05 level of significance. The Kolmogorov Smirnov test statistics values for the six models being investigated are shown in Table 2. All the values of the Kolmogorov Smirnov test statistics are lower than the theoretical critical value, except the Rayleigh distribution. Hence, it is concluded that these fitted models except the Rayleigh distribution to the repair times (in $\mathrm{hr}$ ) for an airborne communication transceiver are adequate. The maximum likelihood estimates of the model parameters, $-2 \mathrm{Ln} \mathrm{L}$, Akaike information criteria (AIC), and Bayesian information criterion (BIC) values for different models are shown in Table 2. It is observed that the RW model has the lowest values of -2Ln L, AIC, and BIC than all the other models and hence the proposed RW model provides a better fitting than all the other models and extensions being considered in the comparison.

Table 1. Repair times (in $\mathrm{h}$ ) for an airborne communication transceiver

\begin{tabular}{lllllllllllllll}
\hline 0.2 & 0.3 & 0.5 & 0.5 & 0.5 & 0.5 & 0.6 & 0.6 & 0.7 & 0.7 & 0.7 & 0.8 & 0.8 & 1.0 & 1.0 \\
\hline 1.0 & 1.0 & 1.1 & 1.3 & 1.5 & 1.5 & 1.5 & 1.5 & 2.0 & 2.0 & 2.2 & 2.5 & 3.0 & 3.0 & 3.3 \\
3.3 & 4.0 & 4.0 & 4.5 & 4.7 & 5.0 & 5.4 & 5.4 & 7.0 & 7.5 & 8.8 & 9.0 & 10.3 & 22.0 & 24.5 \\
\hline
\end{tabular}

Table 2. Results of ML estimates and model evaluation criterion for the data

\begin{tabular}{ccccccc}
\hline Distribution & ML Estimates & Ln L & $-2 \operatorname{lnL}$ & AIC & BIC & K-S \\
\hline Exponential $(\lambda)$ & 0.2757 & -102.97 & 205.94 & 207.94 & 209.8 & 0.173 \\
Rayleigh $(\beta)$ & 0.16313 & -147.70 & 295.40 & 297.40 & 299.2 & 0.457 \\
GE $(\alpha, \lambda)$ & $0.9383,0.2640$ & -102.92 & 205.84 & 209.84 & 213.5 & 0.161 \\
GR $(\alpha, \beta)$ & $0.2872,0.0542$ & -109.36 & 218.36 & 222.36 & 226.3 & 0.180 \\
MR $(\alpha, \beta)$ & $0.002368,0.02661$ & -101.88 & 203.76 & 207.76 & 211.4 & 0.148 \\
RIW $(\beta, \alpha)$ & $1.0558,0.51$ & -98.40 & 196.80 & 200.80 & 204.41 & 0.090 \\
RW $(\beta, \alpha)$ & $0.5858,0.44$ & -88.54 & 177.08 & 181.08 & 184.69 & 0.120 \\
\hline
\end{tabular}

\subsection{Application 2}

The survival times of a group of patients suffering from Head and Neck cancer disease who were treated using a combination of radiotherapy and chemotherapy [24] are considered. Table 3 includes these data. Rama [25] fits exponential, Gamma, and Weibull distribution to the above data set. The maximum likelihood estimates for the model parameters, -2ln L, AIC, BIC, and Kolmogorov-Smirnov test statistic values are shown in Table 4. The proposed models Rayleigh inverted-Weibull (RIW) and Rayleigh weibull distributions (RF) in addition to Rayleigh baseline distribution are used in this work to fit the data. The results are also shown in Table 4. The theoretical critical value of Kolmogorov-Smirnov (K-S) test is $K-S_{0.05}=0.205$ at 0.05 level of significance. All the values of the Kolmogorov Smirnov statistic values are lower than the theoretical critical value, except the Rayleigh distribution. Hence, the adequacy of fitting these models to the survival times data except the Rayleigh distribution is verified. It is observed that the RF model has the lowest values of -2Ln L, AIC, and BIC than exponential, Gamma, Weibull distributions, Rayleigh, and Rayleigh inverted Weibull (RIW) distribution, and hence the proposed Rayleigh Weibull (RW) model provides better fitting than all the other models being considered in the comparison.

Table 3. Survival times of patients suffering from Head and Neck cancer disease

\begin{tabular}{ccccccccccc}
\hline 12.20 & 23.56 & 23.74 & 25.87 & 31.98 & 37 & 41.35 & 47.38 & 55.46 & 58.36 & 63.47 \\
\hline 68.46 & 78.26 & 74.47 & 81.43 & 84 & 92 & 94 & 110 & 112 & 119 & 127 \\
130 & 133 & 140 & 146 & 155 & 159 & 173 & 179 & 194 & 195 & 209 \\
249 & 281 & 319 & 339 & 432 & 469 & 519 & 633 & 725 & 817 & 1776 \\
\hline
\end{tabular}

Table 4. Results of ML estimates and model evaluation criterion for the data

\begin{tabular}{cccccc}
\hline Distribution & ML Estimates & $-2 \operatorname{lnL}$ & AIC & BIC & K-S \\
\hline Exponential $(\lambda)$ & 0.0045 & 564.03 & 566.03 & 567.81 & 0.139 \\
Rayleigh $(\beta)$ & 0.00266 & 643.91 & 645.91 & 651.48 & 0.490 \\
Gamma $(\lambda, \alpha)$ & $0.0064,1.0476$ & 564.03 & 568.03 & 571.60 & 0.148 \\
Weibull $(\lambda, \alpha)$ & $0.0064,0.9404$ & 563.68 & 567.68 & 571.25 & 0.129 \\
RIW $(\beta, \alpha)$ & $1.0558,0.51$ & 559.00 & 563.00 & 566.57 & 0.110 \\
RW $(\beta, \alpha)$ & $0.082,0.47$ & 536.69 & 540.69 & 544.26 & 0.129 \\
\hline
\end{tabular}




\section{CONCLUSION}

Two new extensions of the Rayleigh distributions were proposed and studied, namely Rayleigh inverted-Weibull (RIW) distribution and Rayleigh Weibull (RW). Some fundamental properties of these new extensions are derived. The maximum likelihood estimators along with asymptotic confidence intervals are also presented. Two real data sets were analyzed using the new extensions and compared with other extensions and competing models. The proposed Rayleigh Weibull (RW) distribution gives better fitting than all the other fitted models considered in the comparison.

\section{REFERENCES}

[1] J. C. Ahuja and S. W. Nash, "The generalized Gompertz-Verhulst family of distributions," Sankhyā: The Indian Journal of Statistics, Series A, vol. 29, no. 2, pp. 141-156, 1967.

[2] R. D. Gupta and D. Kundu, "Exponentiated Exponential Family: An alternative to Gamma and Weibull," Biometrical Journal, vol. 33, no. 1, pp. 117-130, 1998, doi: 10.1002/1521-4036(200102)43:1\%3C117: AIDBIMJ117\%3E3.0.CO;2-R.

[3] R. D. Gupta and D. Kundu, "Generalized exponential distributions," Australian and New Zealand Journal of Statistics, vol. 41, no. 2, pp. 173-188, 1999, doi: 10.1111/1467-842X.00072.

[4] P. Kumaraswamy, "Generalized probability density-function for double-bounded random-processes," Journal of Hydrology, vol. 46, no. 1-2, pp. 79-88, 1980, doi: 10.1016/0022-1694(80)90036-0.

[5] A. N. Marshall and I. Olkin, "A new method for adding a parameter to a family of distributions with applications to the exponential and Weibull families," Biometrika, vol. 84, no. 3, pp. 641-652, 1997, doi: 10.1093/biomet/84.3.641.

[6] D. C. T. Granzotto, F. Louzada, and N. Balakrishnan, "Cubic rank transmuted distributions: Inferential issues and applications," Journal of Statistical Computation and Simulation, vol. 87, no. 14, pp. 2760-2778, 2017, doi: 10.1080/00949655.2017.1344239.

[7] A. Alzaatreh, C. Lee, and F. Famoye, "A new method for generating families of continuous distributions," Metron, vol. 71, pp. 63-79, 2013, doi: 10.1007/s40300-013-0007-y.

[8] A. Mahdavi and D. Kundu, "A new method for generating distributions with an application to exponential distribution," Communications in Statistics-Theory and Methods, vol. 46, no. 13, pp. 6543-6557, 2017, doi: 10.1080/03610926.2015.1130839.

[9] G. M. Cordeiro, M. Alizadeh, and R. P. Marinho, "The type I half-logistic family of distributions," Journal of Statistical Computations and Simulations, vol. 86, no. 4, pp. 707-728, 2015, doi: 10.1080/00949655.2015.1031233.

[10] A. S. Hassan, M. Elgarhy, and M. Shakil, "Type II half logistic family of distributions with applications," Pakistan Journal of Statistics and Operations Research, vol. 13, no. 2, pp. 245-264, 2017, doi: 10.18187/pjsor.v13i2.1560.

[11] C. Alexander, G. M. Cordeiro, E. M. Ortega, and J. M. Sarabia, "Generalized beta-generated distributions," Computational Statistics and Data Analysis, vol. 56, no. 6, pp. 1880-1897, 2012, doi: 10.1016/j.csda.2011.11.015.

[12] M. Bourguignon, R. B. Silva, and G. M. Cordeiro, "The Weibull-G family of probability distributions," Journal of Data Science, vol. 12, pp. 53-68, 2014.

[13] J. G. Surles and W. J. Padgett, "Inference for reliability and stress-strength for a scaled Burr Type X distribution," Lifetime Data Analysis, vol. 7, no. 2, 187-200, 2001, doi: 10.1023/A:1011352923990.

[14] A. E. Gomes, C. Q. da-Silva, G. M. Cordeiro, and E. M. M. Ortega, "A new lifetime model: The Kumaraswamy generalized Rayleigh distribution,” Journal of Statistical Computation and Simulation, vol. 84, no. 2, pp. 290-309, 2014, doi: 10.1080/00949655.2012.706813.

[15] K. A. Al-Kadim and B. D. Mohammed, "Rayleigh Pareto distribution," Journal of Babylon University/Pure and Applied Sciences, vol. 26, no. 1, pp. 84-95, 2018.

[16] D. Kundu and M. Z. Raqab, "Generalized Rayleigh distribution: different methods of estimations," Computational Statistics and Data Analysis, vol. 49, no. 1, pp. 187-200, 2005, doi: 10.1016/j.csda.2004.05.008.

[17] N. Saudi and C. Sohail, "Modified Rayleigh distribution," Conference Paper: 16th international conference on statistical sciences: Advances in Statistics and Data Management: Its Role in National Growth and SocioEconomic Developments, Peshawar, Pakistan, March 2018.

[18] A. A. Al-Babtain, "A new extended Rayleigh distribution," Journal of King Saud University Science Journal, vol. 32, no. 5, pp. 2576-2581, Jul. 2020, doi: 10.1016/j.jksus.2020.04.015.

[19] M. Ganji, H. Bevrani, N. H. Golzar, and S. Zabihi, "The Weibull-Rayleigh distribution, Some Properties, and Applications," Journal of Mathematical Sciences, vol. 218, no. 3, pp. 269-277, 2016.

[20] D. Bahati, M. A. Malik, and H. J. Vaman, "Lindley-exponential distribution: Properties and applications," Metron, vol. 73, no. 3, pp. 335-357, Dec. 2015, doi: 10.1007/s40300-015-0060-9.

[21] N. Ekhosuehi and F. Opone, "Three parameter generalized Lindley distribution: properties and applications," Statistica, vol. 78, no. 3, pp. 233-249, 2018, doi: 10.6092/issn.1973-2201/8123.

[22] J. J. Moors, "A quantile alternative for kurtosis," Journal of the Royal Statistical Society, Series D (Statistician), vol. 37, no. 1, pp. 25-32, 1988, doi: 10.2307/2348376.

[23] R. D. Gupta and D. Kundu, "Generalized exponential distribution: different method of estimations," Journal of Statistical Computation and Simulation, vol. 69, no. 4, pp. 315-337, 2001, doi: 10.1080/00949650108812098.

[24] B. Efron, "Logistic regression, survival analysis and the Kaplan-Meier curve," Journal of the American Statistical Association, vol. 83, no. 402, pp. 414- 425, 1988, doi: 10.1080/01621459.1988.10478612. 
[25] S. Rama, K. K. Shukla, S. Ravi, and Leonida T. A., "On modeling of lifetime data using two-parameter gamma and Weibull distributions," Biometrics and Biostatistics International Journal, vol. 3, no. 2, pp. 201-206, 2016, doi: 10.15406/bbij.2016.03.00061.

\section{BIOGRAPHIES OF AUTHORS}



Mahmoud M. Smadi, received his B.S and M.S degrees in statistics from Yarmouk University in 1983 and 1986, He received his Ph. D in statistics from Colorado State University in 1997. Since 1997 he worked for the Department of Mathematics \& Statistics at Jordan University of Science \& Technology, Jordan. He is currently associate professor at Department of Mathematics \& Statistics at Jordan University of Science \& Technology. His research interests include survival and reliability analysis, biostatistical applications.

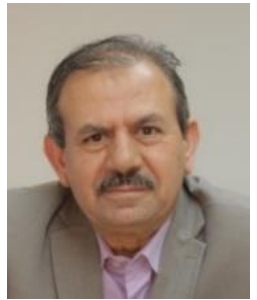

Mahmoud Alrefaei is a professor of operations research at Jordan University of Science and Technology (JUST), Jordan. He received his Ph.D. in Mathematics and Industrial Engineering from the University of Wisconsin-Madison, USA in 1997. He was a research assistant at Georgia Institute of Technology from 1995 to 1997 . He joined the Mathematics and Statistics Department at JUST in 1997. He then joined Qatar University from 2006 to 2012. His research interest includes Simulated Annealing; Multi-Objective Simulation; Supply Chain Management; Stochastic Optimization; Statistical Selection and Ordinal Optimization. He has published more than 50 publications in international reputable journals. Further information can be found on his homepage: http://www.just.edu.jo/eportfolio/Pages/Default.aspx?email=alrefaei. 